\title{
Taspase1 cleaves MLL1 to activate cyclin E for HER2/neu breast tumorigenesis
}

\author{
Yiyu Dong ${ }^{1, *}$, Brian A Van Tine ${ }^{5, *}$, Toshinao Oyama ${ }^{1}$, Patricia I Wang ${ }^{1}$, Emily H Cheng ${ }^{1,2}$, James J Hsieh ${ }^{1,3,4}$ \\ ${ }^{1}$ Human Oncology \& Pathogenesis Program, Memorial Sloan-Kettering Cancer Center, New York, NY 10065, USA; ${ }^{2}$ Depart- \\ ment of Pathology, Memorial Sloan-Kettering Cancer Center, New York, NY 10065, USA; ${ }^{3}$ Department of Medicine, Memorial \\ Sloan-Kettering Cancer Center, New York, NY 10065, USA; ${ }^{4}$ Department of Medicine, Weill Cornell Medical College, New York, \\ NY 10021, USA; '5epartment of Internal Medicine, Washington University, St Louis, MO 63110, USA
}

Taspase1, a highly conserved threonine protease, cleaves nuclear transcriptional regulators mixed-lineage leukemia (MLL, MLL1), MLL2, TFIIA, and ALF to orchestrate a wide variety of biological processes. In vitro studies thus far demonstrated that Taspase1 plays important roles in the proliferation of various cancer cell lines, including HER2-positive breast cancer cells. To investigate the role of Taspase1 in breast tumorigenesis in vivo, we deleted Taspase1 from mouse mammary glands by generating MMTV-neu;MMTV-cre;Tasp $1^{F /}$ mice. We demonstrate that initiation of $M M T V$-neu- but not $M M T V$-wnt-driven breast cancer is blocked in the absence of Taspase1. Importantly, Taspase1 loss alone neither impacts normal development nor pregnancy physiology of the mammary gland. In mammary glands Taspase1 deficiency abrogates MMTV-neu-induced cyclins $\mathbf{E}$ and A expression, thereby preventing tumorigenesis. The mechanisms were explored in HER2-positive breast cancer cell line BT474 and HER2-transformed MCF10A cells and validated using knockdown-resistant Taspase1. As Taspase1 was shown to cleave MLL which forms complexes with E2F transcription factors to regulate Cyclins $E, A$, and $B$ expression in mouse embryonic fibroblasts (MEFs), we investigated whether the cleavage of MLL by Taspase1 constitutes an essential in vivo axis for HER2/neu-induced mammary tumorigenesis. To this end, we generated $M M T V-n e u ; M L L^{n c / n c}$ transgenic mice that carry homozygous non-cleavable $M L L$ alleles. Remarkably, these mice are also protected from HER2/neu-driven breast tumorigenesis. Hence, MLL is the primary Taspase1 substrate whose cleavage is required for MMTV-neuinduced tumor formation. As Taspase1 plays critical roles in breast cancer pathology, it may serve as a therapeutic target for HER2-positive human breast cancer.

Keywords: Taspase1; MLL; cyclin E; HER2; breast cancer

Cell Research (2014) 24:1354-1366. doi:10.1038/cr.2014.129; published online 30 September 2014

\section{Introduction}

Human breast cancer is a heterogeneous disease comprised of three major subgroups, each encompassing unique molecular signatures, prognoses, and responses to therapies [1]. HER2 is a member of the epidermal growth factor receptor (EGFR) family of receptor tyrosine kinases, which includes EGFR, HER2, HER3, and HER4 $[2,3]$. Homo- or hetero-dimerization of these re-

\footnotetext{
*These two authors contributed equally to this work. Correspondence: James J Hsieh

E-mail: hsiehj@mskcc.org

Received 24 April 2014; revised 29 July 2014; accepted 17 August 2014; published online 30 September 2014
}

ceptors results in the phosphorylation of residues in the intracellular domain and the consequent recruitment of adapter molecules responsible for the initiation of several signaling pathways involved in cell proliferation and survival. HER2-amplified/overexpressed breast cancer is one of the aforementioned subgroups. It accounts for approximately $20 \%-30 \%$ of all breast cancer cases, and it is characterized by an aggressive phenotype and poor overall survival [4-6]. Despite therapeutic advances brought forth by anti-HER2 agents including monoclonal antibodies such as trastuzumab (Herceptin) and small molecule tyrosine kinase inhibitors, patients with advanced HER2-positive breast cancer frequently experience disease progression and/or recurrence [7-9].

One of the hallmarks of cancer is dysregulated prolif- 
eration [10]. Unsurprisingly, molecular characterization of human tumors reveals that key cell cycle regulators are frequently dysregulated [11]. During cell cycle progression, cyclin-dependent kinases (CDKs) and cyclins constitute the central regulatory apparatus. In mammalian cells, kinase subunits (CDK4, CDK6, CDK2, and $\mathrm{CDC} 2$ ) are expressed alongside cyclins (cyclin D, E, $\mathrm{A}$, and B) sequentially as the cells progress from G1 through mitosis. CDK4 and CDK6 form complexes with one of several D-type cyclins and function early in G1 phase, probably in response to growth factors. CDK2 forms complexes with cyclins $\mathrm{E}$ or $\mathrm{A}$ and functions in the G1/S phase transition and S phase DNA replication [1214]. A major molecular consequence of HER 2 up-regulation is the increased expression of $\mathrm{G}_{1}$-to-S cell cycle regulatory proteins cyclins $\mathrm{D}$ and $\mathrm{E}$, which leads to aberrant cell proliferation [7, 12-16]. High cyclin E expression is a marker that correlates strongly with poor outcome in patients with breast cancer. Since Cyclin E amplification/ overexpression leads to trastuzumab resistance, disrupting Cyclin E expression could have therapeutic importance for HER2-positive breast cancers [17, 18].

Taspase 1 was originally purified as the protease that cleaves MLL (the Mixed-Lineage Leukemia protein; also known as MLL1) for proper regulation of $H O X$ gene expression $[19,20]$. Other genetically and biochemically proven Taspase1 substrates include MLL2 (also known as MLL4), TFIIA $\alpha-\beta$, ALF $\alpha-\beta$ (TFIIA-Like Factor) and Drosophila HCF-1 ( estingly, all confirmed Taspase1 substrates are nuclear transcription factors that play important roles in gene regulation. Taspasel encodes a highly conserved $50 \mathrm{kDa}$ $\alpha-\beta$ proenzyme, which undergoes intramolecular autoproteolysis, producing the mature $\alpha 28 / \beta 22$ heterodimeric enzyme that displays an overall $\alpha / \beta / \beta / \alpha$ structure [20, 25]. A complete genetic knockout of Taspasel in mice resulted in profound early postnatal lethality and the few surviving Taspase ${ }^{-/}$mice universally exhibited small body sizes and homeotic transformations at the axial skeleton [22]. Taspaes $1^{-/}$mouse embryonic fibroblasts (MEFs) displayed cell cycle progression defects with downregulation of cyclins $E, A$, and $B$ and upregulation of $C D K I s$ (cyclin-dependent kinase inhibitors) $p 16$, $p 21$ and $p 27$ [22]. Importantly, Taspase $1^{-/}$MEFs were resistant to oncogenic transformation in vitro [26]. In MEFs, Taspase1 cleaves MLL that interacts with E2Fs, core transcription factors of the mammalian cell cycle, to activate select Cyclin genes [22, 27, 28]. How Taspase1 regulates $C D K I s$, however, remains unclear. Importantly, Taspase 1 shows a high level of expression in most human cancer cell lines [22], and knockdown of Taspase1 in many cancer cell lines impairs cancer cell proliferation and even sensitizes brain cancer and melanoma cells to anoikis [26].

Encouragingly, despite the fact that Taspase1 plays an important role in mammalian embryogenesis, acute genetic deletion of Taspase 1 in adult mice does not confer discernible toxicities on the mice, which suggests a wide therapeutic index for Taspase 1 inhibition in adult cancer patients [29]. Moreover, pharmacological inhibition of Taspase 1 has been attempted [29, 30], and a primitive small molecular Taspase1 inhibitor (TASPIN) showed effects on U251 brain tumor xenografts and HER2-driven mouse breast cancers [29]. The latter findings prompted us to hypothesize that Taspase1 could play a critical role in HER2-positive breast cancer and that Taspase1 inhibitors may be developed as a safe treatment option for Taspase1-dependent cancers.

Here, we report the data from preclinical experiments that we conducted by constructing genetically well-defined mouse models, demonstrating that Taspase1 ablation blocks MMTV-neu-driven breast cancer initiation in vivo. We further pinpoint that MLL is the key Taspase 1 substrate whose cleavage is required for MMTV-neuinduced tumor formation. The cleavage of MLL by Taspase1 enables HER2/neu-induced overexpression of Cyclins $E$ and $A$, presenting an essential in vivo genetic network conferring breast tumorigenesis.

\section{Results}

Taspase1 deficiency disrupts the expression of cyclins and proliferation of $\mathrm{HER}^{+}$breast cancer cells

To determine whether Taspase 1 is required for HER2-positive breast cancer cell proliferation, we conducted genetic knockdown experiments in two HER2-overexpressing breast cancer cell lines, BT474 and HCC1419. Taspase1 deficiency significantly reduced the cell number in both cell lines (Figure 1A). Cell death assay confirmed that there is no significant difference in cell death between the Taspase 1 knockdown cells and the control in either cell line (Figure 1B). On the other hand, cell cycle analysis showed that Taspase 1 knockdown significantly decreased the $\mathrm{S}$ phase population in both cell lines (Figure 1C). These data suggest that Taspase1 regulates HER2-positive breast cancer cell proliferation through promoting cell cycle progression.

We next investigated the underlying mechanisms by which Taspase 1 regulates cell division. Key regulators of the mammalian cell cycle machinery include E2Fs, Rbs, cyclins, cyclin-dependent kinases (CDKs), and CDK inhibitors (CDKIs), which form complex positive and negative epistatic regulatory loops to ensure accurate cell cycle progression. In MEFs, following the cleav- 
A

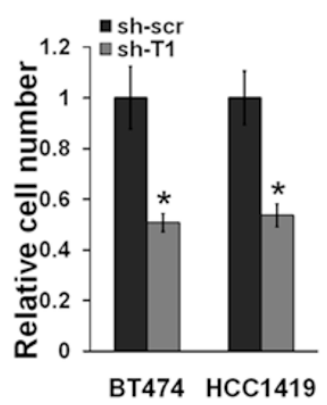

B

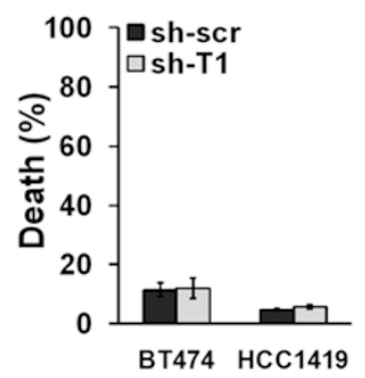

C

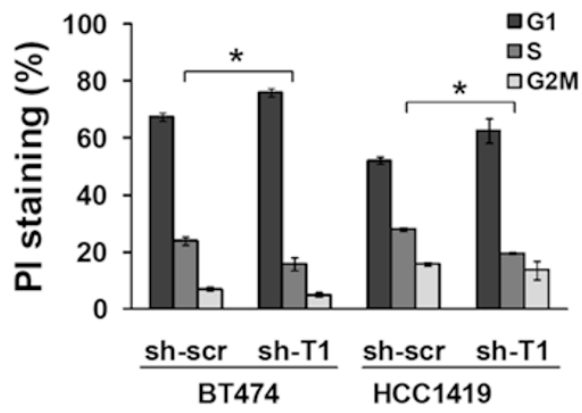

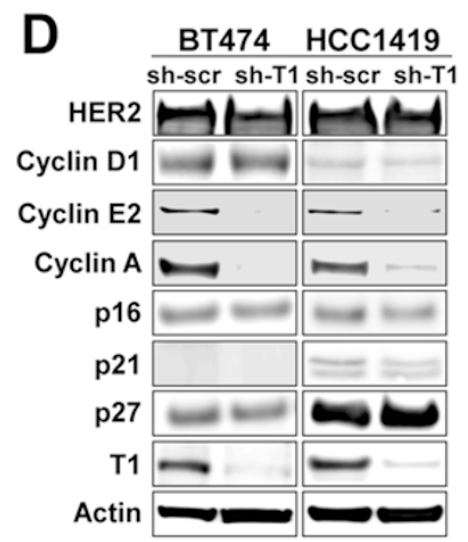

$\mathbf{F}$

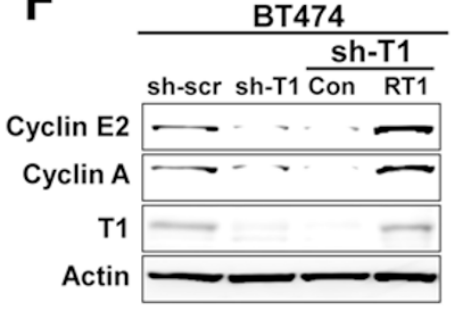

E

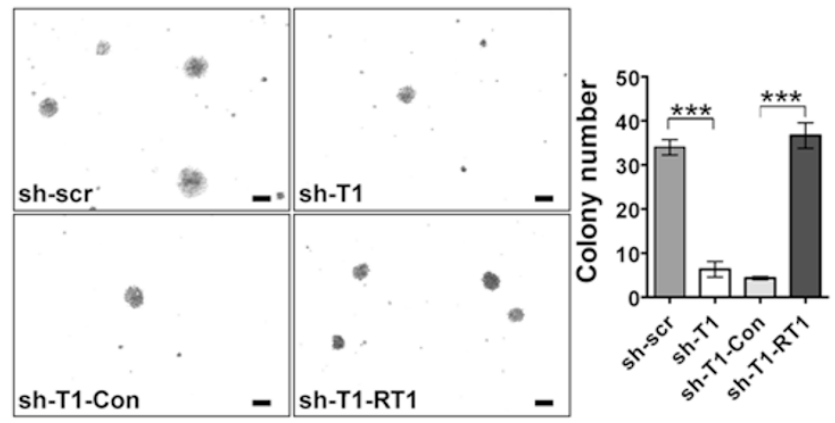

G

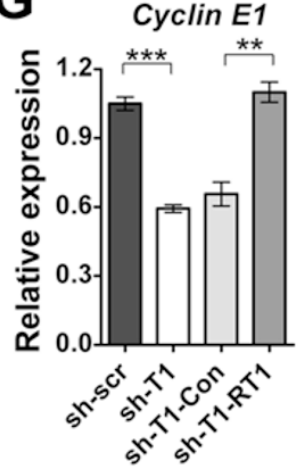

Cyclin E2

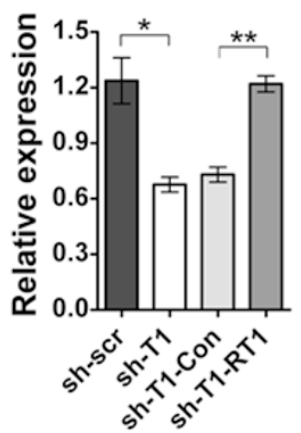

Cyclin A

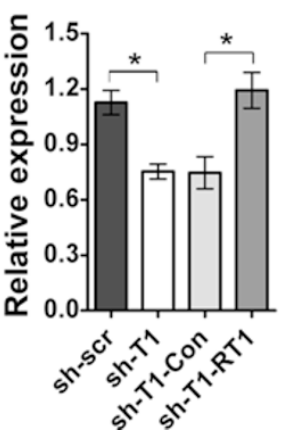

Figure 1 Taspase1 deficiency disrupts the proliferation of HER2-positive breast cancer cells. (A) Proliferation of Taspase1 knockdown BT474 and HCC1419 cells. $1 \times 10^{5}$ scramble-control (sh-scr) or Taspase1 (sh-T1) knockdown cells were seeded in triplicate wells and counted at day 4. Data presented are mean \pm SD of three independent experiments. ${ }^{*} P<0.001$. (B) Apoptosis of Taspase1 knockdown BT474 and HCC1419 cells. Cell death of scramble-control (sh-scr) or Taspase1 (sh-T1) knockdown cells was assessed by FACS analysis, following Annexin V staining. Data presented mean \pm SD of four independent experiments. (C) Cell cycle profiles of Taspase1 knockdown BT474 and HCC1419 cells. The indicated scramble-control (sh-scr) or Taspase1 (sh-T1) knockdown cells were stained with propidium iodide (PI) and analyzed by FACS. Data presented are mean \pm SD of four independent experiments. ${ }^{*} P<0.005$. (D) Western blots of cyclins, CDKIs, and Taspase1 on scramble-control (sh-scr) or Taspase1 (sh-T1) knockdown BT474 and HCC1419 cells. $\beta$-Actin serves as loading control. (E) Soft agar assays using Taspase1 knockdown BT474 cells expressing either empty vector (sh-T1-Con) or knockdown resistant Taspase1 (sh-T1-RT1). Scale bar, $200 \mu \mathrm{m}$. Data presented are the mean \pm SD. ${ }^{* *} P<0.0005$. (F) Western blots of cyclin E2, cyclin A, Taspase1, and $\beta$-Actin on scramble-control knockdown (sh-scr), Taspase1 knockdown (sh-T1), Taspase1 knockdown expressing empty vector (sh-T1-Con), and Taspase1 knockdown expressing knockdown resistant Taspase1 (sh-T1RT1) BT474 cells. (G) qRT-PCR analyses of scramble-control knockdown (sh-scr), Taspase1 knockdown (sh-T1), Taspase1 knockdown expressing empty vector (sh-T1-Con), and Taspase1 knockdown expressing knockdown resistant Taspase1 (shT1-RT1) BT474 cells. Expression was normalized to human $18 S r R N A$. Data presented are mean \pm SD of three independent experiments. ${ }^{\star} P<0.05 ;{ }^{* *} P<0.005 ;{ }^{* *} P<0.0005$. 
age by Taspase1, MLL ${ }^{\mathrm{N} 320 / \mathrm{C} 180} \operatorname{targets}$ to Cyclins $E$ and $A$ promoters through interaction with E2Fs to methylate histone $\mathrm{H} 3$ at K4, thereby transactivating Cyclins $E$ and $A$ for cell proliferation $[22,28]$. To gain mechanistic insight into how Taspase1 regulates HER2-positive breast cancer cell proliferation, we examined the expression of several key cell cycle regulators. Western blot analyses of Taspase1-knockdown BT474 and HCC1419 cells revealed a significant decrease in cyclins $\mathrm{E} 2$ and $\mathrm{A}$, but not D1 (Figure 1D), consistent with our prior results obtained in MEFs [22]. Altogether, these results indicate that in HER2-positive breast cancer cells Taspase1 assures the proper accumulation of cyclins $\mathrm{E}$ and $\mathrm{A}$ for proliferation.

The ability of cancer cells to form colonies on soft agar is a stringent in vitro surrogate of in vivo tumorigenicity. Soft agar assays assess the capacity of tumor cells to not only proliferate but also resist anoikis under three-dimensional culture conditions that imitate the in vivo tumor growth environment. We determined the degree to which Taspase1 is required for the colony formation capability of HER2-positive breast cancer cells on soft agar. Knockdown of Taspase1 (sh-T1) in BT474 cells severely compromised their ability to grow as colonies on soft agar (Figure 1E). To validate the specific requirement of Taspase 1 for cancer cell growth on soft agar, we engineered a sh-T1 knockdown resistant version of Taspase1 (RT1). Retroviral delivery of RT1 rescued the ability of Taspase1-knockdown (sh-T1) BT474 cells to form colonies (Figure 1E). Western blot analysis confirmed the successful knockdown of Taspase1 in BT474 cells by sh-T1 and the resulting reduced protein levels of cyclins E2 and A (Figure 1F), and the resistance of RT1 to sh-T1 and the restoration of the protein levels of cyclins E2 and A in RT1-reconstituted sh-T1 BT474 cells (Figure 1F). We further interrogated the mechanisms by which Taspase1 sustains cyclins levels. Quantitative real-time PCR (qRT-PCR) assays revealed that the mRNA levels of Cyclins E1,E2, and $A$ were reduced in sh-T1 BT474 cells and were restored to baseline in RT1 sh-T1 BT474 cells (Figure 1G). Similar results were obtained utilizing HER2-transformed MCF10A cells (Supplementary information, Figure S1A and S1B). Altogether, these results indicate that Taspase 1 controls the cell division cycle of HER2-positive breast cancer cells largely by conferring proper transcription of the Cyclins $E$ and $A$ genes upon aberrant receptor tyrosine kinase signaling.

Deletion of Taspase1 in mouse mammary glands blocks MMTV-neu-driven breast cancer formation

To determine whether Taspase 1 is required for breast tumorigenesis in vivo, we generated MMTV-neu; $M M T V$ - cre; $\operatorname{Tasp} 1^{F /-}$ mice by employing the widely adapted MMTV-neu mouse model [15, 31]. MMTV-neu;MMTVcre;Tasp $1^{+/+}$female mice were generated as positive controls and monitored for breast cancer formation. All of our MMTV-neu; MMTV-cre; $T a s p 1^{+/+}$virgin female mice $(n$ $=30$ ) developed breast tumors between 30 and 50 weeks of age (Figure 2A, 2B), as did their counterparts in previously published studies $[15,31]$. In stark contrast, 26 of 30 MMTV-neu;MMTV-cre;Tasp $1^{F /}$ females remained breast cancer free at 60 weeks of age (Figure 2A). Notably, western blot analysis of 12 week-old mammary glands detected similar levels of HER2/neu protein expression in both MMTV-neu;MMTV-cre;Tasp ${ }^{+/+}$and $M M T V$-neu;MMTV-cre;Tasp $1^{F /-}$ female virgin mice (Supplementary information, Figure S2). Interestingly, Taspase 1 ablation did not block the tumor initiation in $M M T V-W n t ; M M T V$-cre;Tasp $1^{F /-}$ female mice, which suggests the specificity of Taspase1 function in HER2-driven breast cancer (Figure 2C-2E). Altogether, these in vivo data in conjunction with our in vitro assays unequivocally establish an essential role for Taspase1 in HER2/ $n e u$-induced breast tumorigenesis in mice and probably in humans.

Genetic deletion of Taspase1 impacts neither normal development nor physiological proliferation of mouse mammary glands

We next investigated the mechanisms by which loss of Taspase 1 in the mammary gland protects MMTV-neu female mice from developing breast cancer by determining whether Taspase 1 ablation simply disrupts mammary gland development and/or response to physiological proliferation signals such as pregnancy. To this end, we compared whole mounts of dissected, carmine-stained mammary glands from MMTV-cre; Tasp $1^{+/+}$and MMTVcre; Tasp $1^{F-}$ female mice, prepared at 6 weeks of age, day 13 of pregnancy, and day 1 of lactation. No macroscopic differences were detected between MMTVcre;Tasp $1^{+/+}$and MMTV-cre;Tasp $1^{F /-}$ mice at any of these pre-specified developmental and physiological states (Figure 3 and Supplementary information, Figure S3). Thus, ablation of Taspase 1 in mouse mammary tissues using MMTV-cre has no effect on normal development or physiological proliferation of mammary glands. Of note, the importance of Taspase 1 in breast tumorigenesis (Figure $2 \mathrm{~A}$ ) and its dispensability in normal mammary gland physiology (Figure 3 and Supplementary information, Figure S3) are reminiscent of those reported with Cyclin D1 knockout mice [15].

Taspase1 deficiency prevents tumor formation of MMTVneu mammary glands 

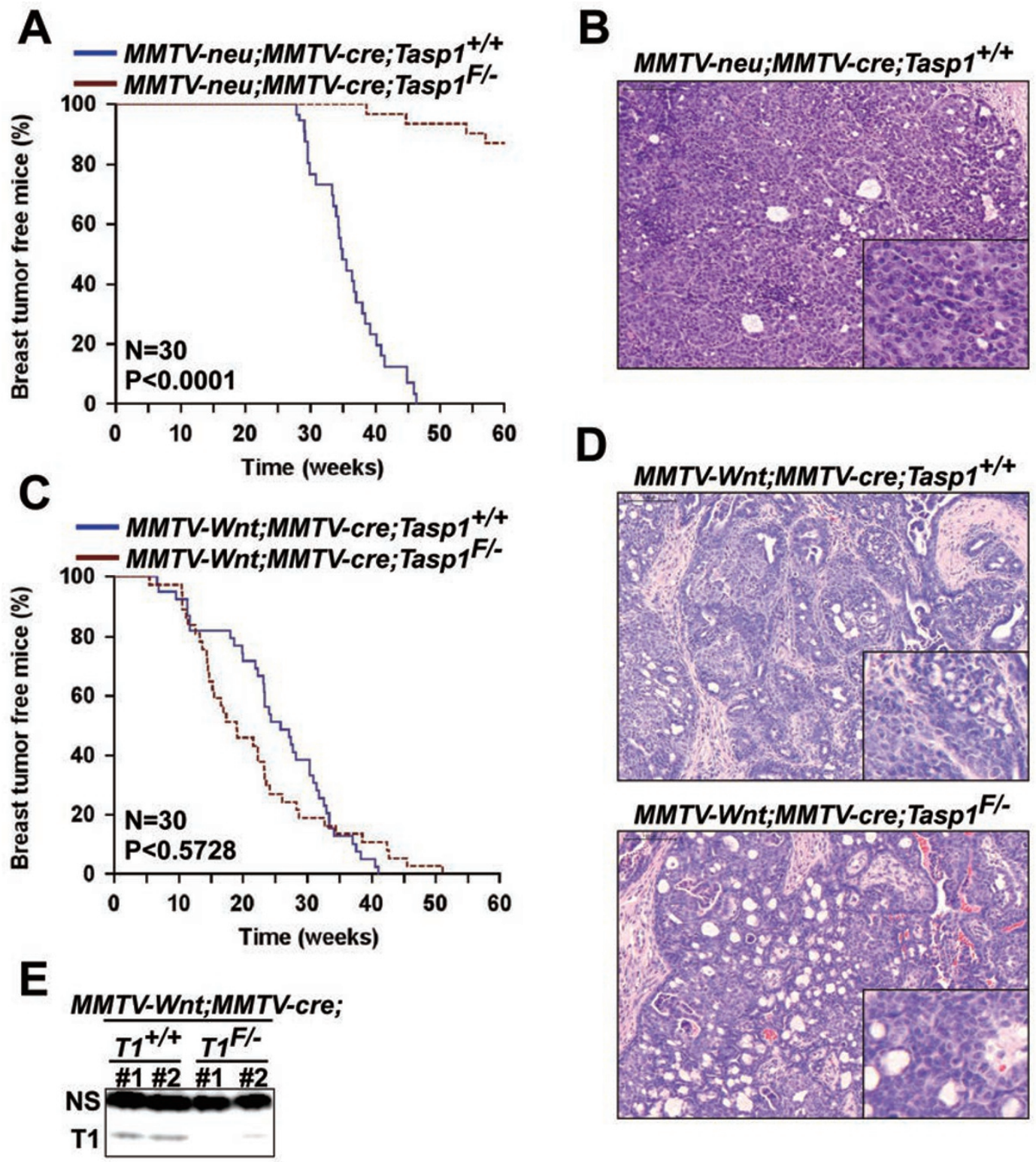

Figure 2 Deletion of Taspase1 in the mammary gland protects mice from developing MMTV-neu-driven breast cancer. (A) Kaplan-Meier curve of breast cancer incidence of MMTV-neu;MMTV-cre;Tasp $1^{\mathrm{F} /}$ female mice. MMTV-neu;MMTV-cre;Tasp $1^{+/+}$ female mice were used as control. $n=30$ mice per genotype. $P<0.0001$. (B) H\&E staining of mouse mammary tumors for the indicated genotypes. Scale bar, $100 \mu \mathrm{m}$. (C) Kaplan-Meier curve of breast cancer incidence of MMTV-Wnt;MMTVcre; Tasp $1^{F /}$ female mice. MMTV-Wnt;MMTV-cre;Tasp $1^{+/+}$female mice were used as control. $n=30$ mice per genotype. $P=$ 0.5728. (D) H\&E staining of mouse mammary tumors for the indicated genotypes. Scale bar, $100 \mu \mathrm{m}$. (E) Western blot analyses of Taspase 1 using female mammary glands of the indicated genotypes. NS indicates non-specific band, serving as control for equal loading.

We analyzed mammary glands of transgenic mice to further dissect the mechanisms by which Taspase1 enables MMTV-neu-induced breast tumorigenesis. Whole mounts were performed using mammary glands of 12-week-old wild-type, MMTV-neu;MMTV-cre;Tasp $1^{+/+}$, and MMTV-neu;MMTV-cre;Tasp $1^{F /}$ female mice to eval- uate glandular structures. In comparison to those of wildtype control mice, mammary glands of $M M T V-n e u ; M$ $M T V$-cre;Tasp $1^{+/+}$mice displayed increased densities in ducts and end buds (Figure 4A), similar to what was previously documented for this mouse strain [31]. Interestingly, this overproliferation phenotype induced by the 


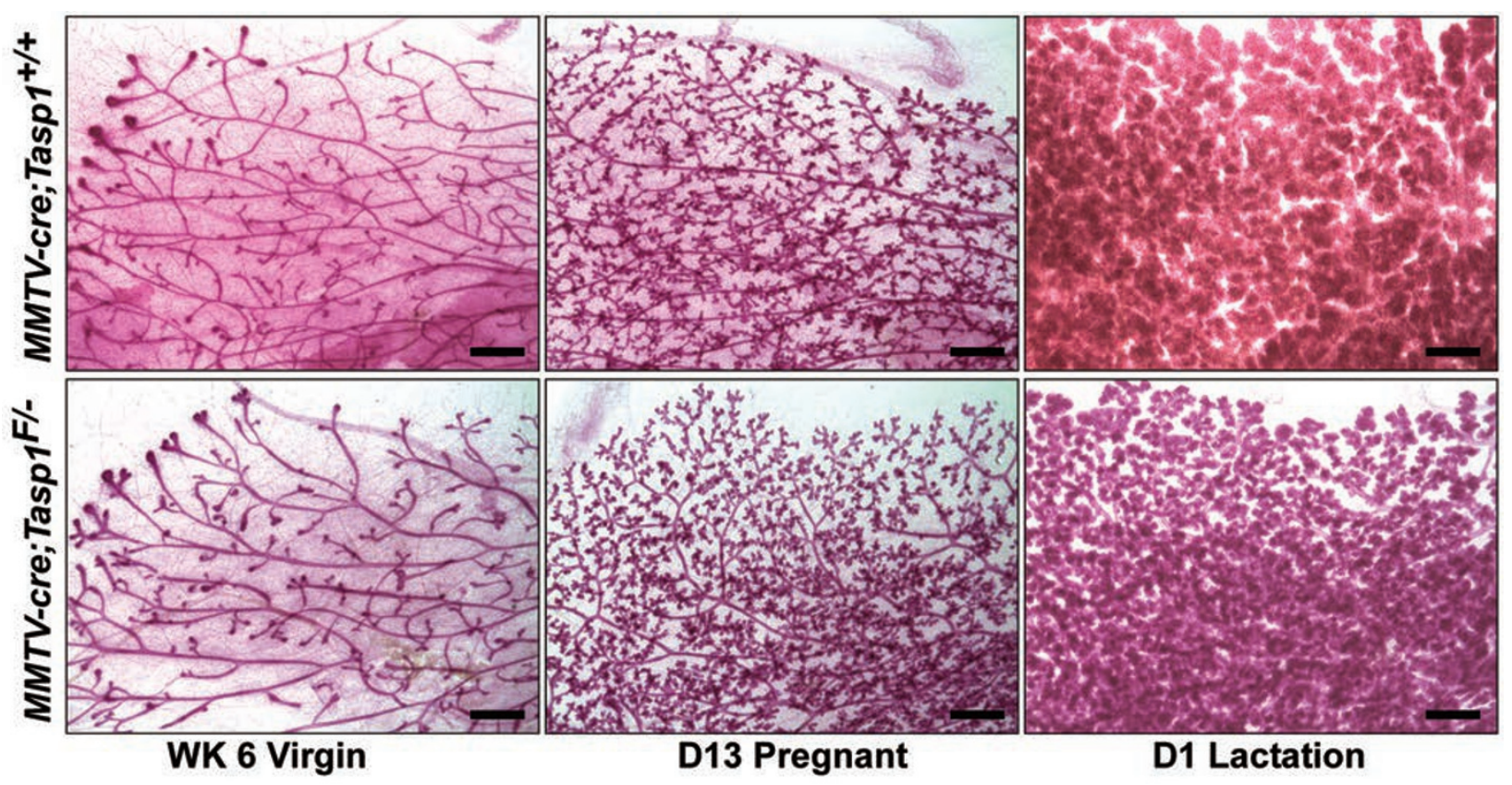

Figure 3 Genetic deletion of Taspase1 does not disrupt mammary gland development or its proliferative response during gestation. Whole mounts of dissected, carmine-stained female mammary glands from mice of the indicated genotypes, age, and gestation status. $n=3-4$ mice per each genotypes. Representative images are presented. Scale bar, $1 \mathrm{~mm}$.

MMTV-neu transgene was not observed in the mammary glands of MMTV-neu;MMTV-cre; Tasp $1^{F /-}$ mice (Figure 4A). Immunohistochemistry for histone H3 serine 10 phosphorylation ( $\mathrm{pH} 3 \mathrm{~S} 10)$ which is a cell proliferation marker, showed that mammary glands of 12-week-old MMTV-neu;MMTV-cre;Tasp $1^{+++}$mice had greater than 10 -fold more $\mathrm{pH} 3 \mathrm{~S} 10$ positive cells than those of Wildtype and $M M T V$-neu;MMTV-cre;Tasp $1^{F /-}$ mice (Figure 4B). Altogether, these genetic data indicate that Taspase1 is required for the $M M T V$-neu-driven aberrant gland proliferation and thus breast carcinogenesis in mice.

$M M T V-n e u$ mice can develop multiple microscopic mammary gland tumors at as early as 14 weeks of age [31]. Accordingly, we examined mammary glands of 20-week-old wild-type, MMTV-neu;MMTVcre;Tasp $1^{+++}$, and MMTV-neu;MMTV-cre;Tasp $1^{F /-}$ female virgin mice by whole mounts. The mammary glands of $M M T V$-neu;MMTV-cre;Tasp1 $1^{+/+}$mice showed significant glandular proliferation and developed tumor foci of various sizes, whereas those of MMTV-neu;MMTVcre;Tasp $1^{F /}$ mice displayed neither overproliferation nor tumor foci, which are macroscopically indistinguishable from wild-type (Figure 4C and Supplementary information, Figure S4). Western blot analysis of 10-weekold wild-type, MMTV-neu;MMTV-cre;Tasp1 ${ }^{+/+}$, and $M M T V$-neu;MMTV-cre;Tasp $1^{F /}$ mammary glands for cyclins E, A, and D, and CDKIs p16 and p27 detect- ed consistent overexpression of cyclin E in $M M T V$ neu; $M M T V$-cre; Tasp $^{+/+}$mice (Figure 4D). A higher level of Taspase1 expression was also detected in these MMTV-neu;MMTV-cre;Tasp $1^{+++}$mammary glands, which is consistent with our prior observation that Taspase 1 is commonly overexpressed in cancer cells [26]. Moreover, examination of $M M T V$-neu; $M M T V$-cre; Tasp $1^{+/+}$breast tumors revealed that cyclins E, A, D, and Taspase1 were all expressed at high abundance (Figure 4D). The successful deletion of Taspase 1 was observed in the MMTVneu;MMTV-cre;Tasp $1^{F-}$ mammary glands and remarkably, MMTV-neu was no longer able to induce cyclin E overexpression in these glands (Figure 4D). These data support the notion that overexpression of cyclin $\mathrm{E}$ and Taspase1 occurs early in MMTV-neu-driven breast tumorigenesis and precedes the overexpression of cyclins A and D.

Taspase1-mediated proteolytic cleavage of MLL is required for MMTV-neu-driven breast cancer formation but dispensable for mammary gland development

Our biochemical and genetic data thus far suggest that Taspase1 enables HER2/neu-driven breast tumorigenesis by permitting transcriptional activation of Cyclin $E$ (Figures 1-4). Since Taspase1 cleaves nuclear factors MLL, MLL2, TFIIA, and ALF to regulate transcriptional programs, we asked the cleavage of which substrate(s) 


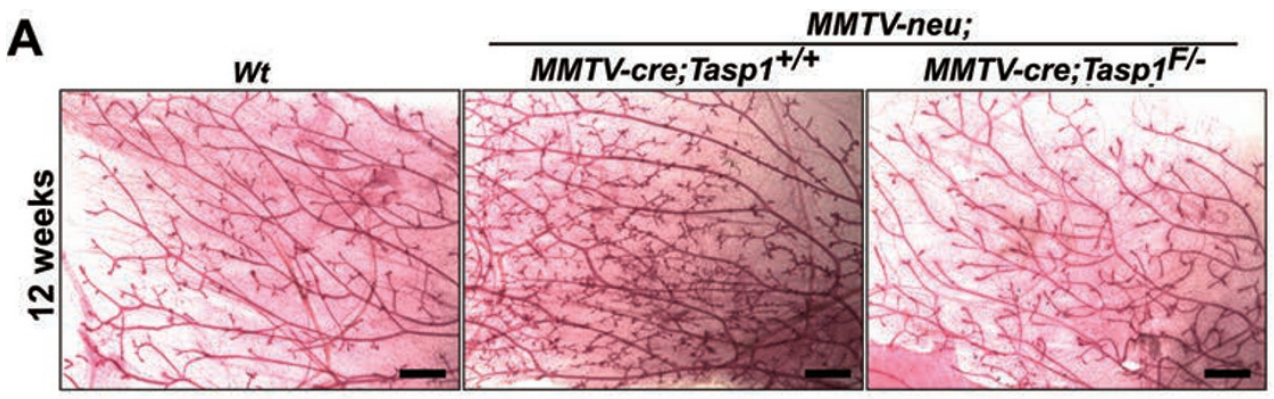

B
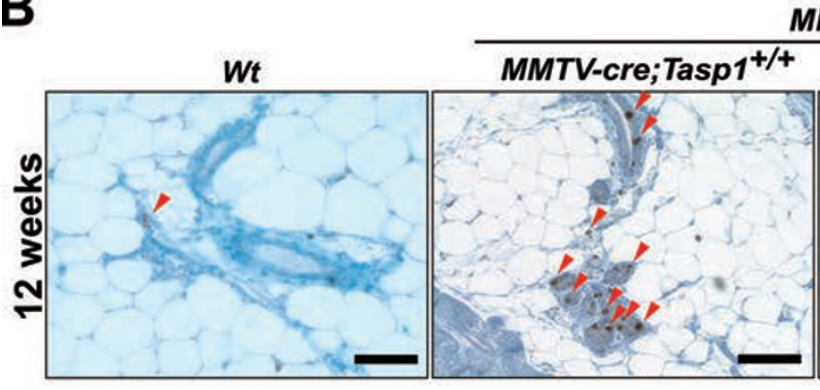

MMTV-neu;

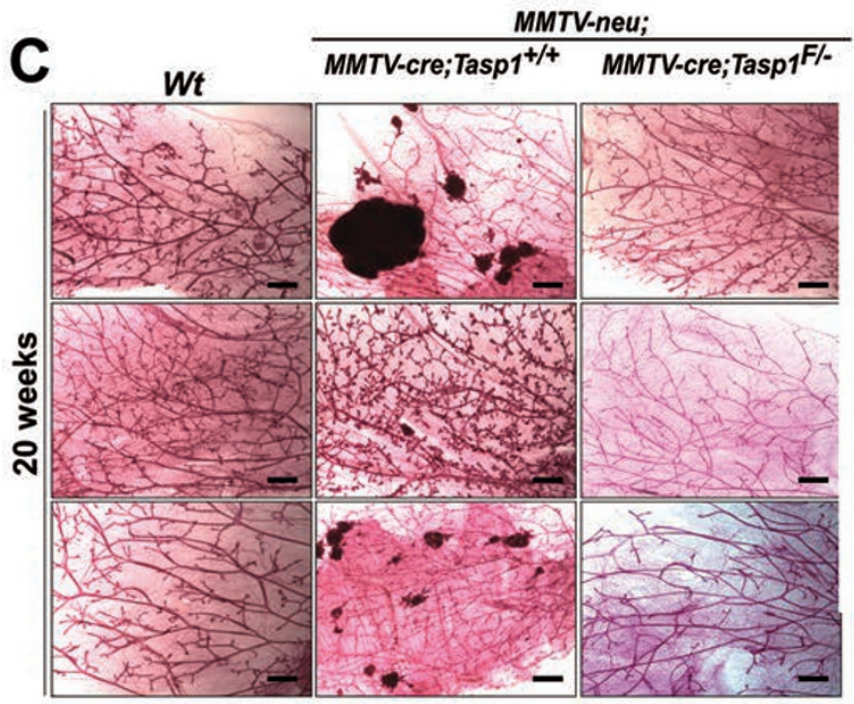

D

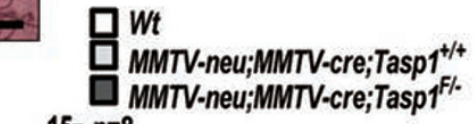
MMTV-cre;Tasp1F/-
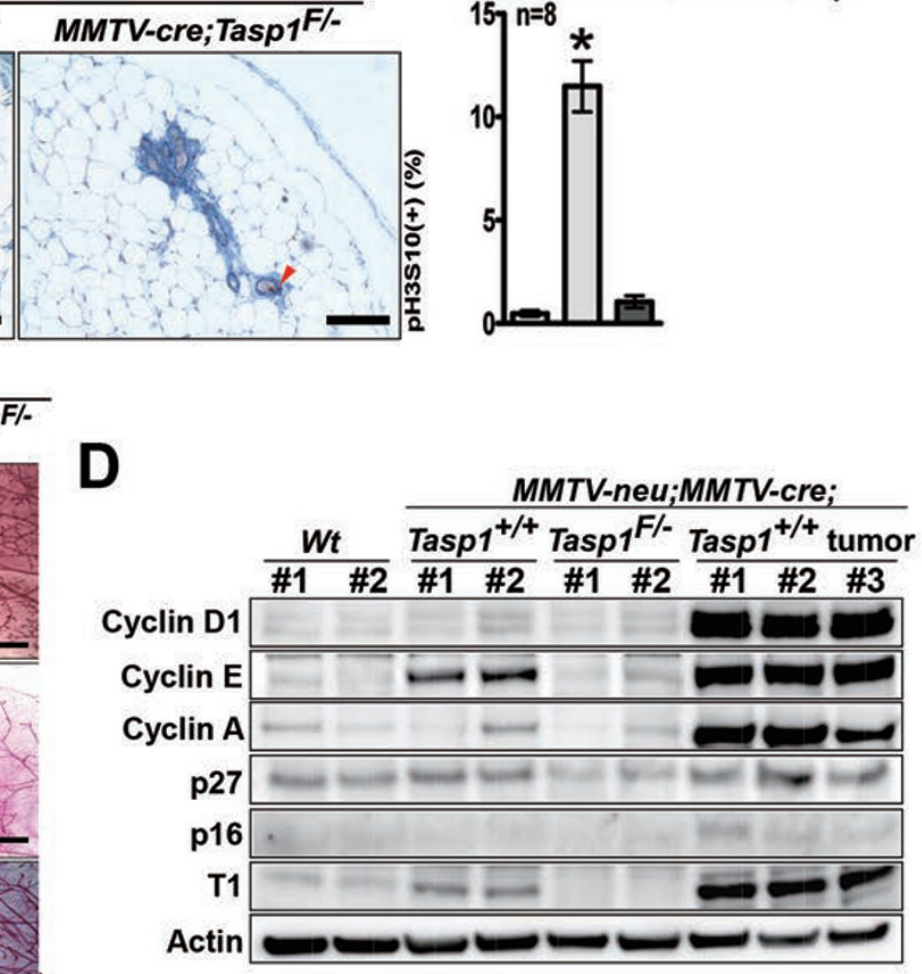

Figure 4 Loss of Taspase1 disrupts the overexpression of cyclin E and the cancer formation in MMTV-neu mouse mammary glands. (A) Whole mounts of dissected, carmine-stained female mammary glands of the indicated genotypes at 12 weeks of age. $n=5-15$ mice per genotype. Representative images are presented. Scale bar, $1 \mathrm{~mm}$. (B) pH3S10 staining of female mammary glands of the indicated genotypes at 12 weeks of age. $n=8$ mammary glands per group. Representative images are presented. Red arrow head indicates positive cells. Scale bar, $100 \mu \mathrm{m}$. ${ }^{*} P<0.0001$. (C) Whole mounts of female mammary glands of the indicated genotypes at 20 weeks of age. $n=5-15$ mice per group. Representative images are presented. Scale bar, $1 \mathrm{~mm}$. (D) Western blot analyses of cyclins, CDKIs, and Taspase1 using female mammary glands and tumor extracts of the indicated genotypes. $\beta$-Actin serves as loading control.

by Taspase 1 is necessary for $M M T V$-neu-induced breast carcinogenesis. Of note, our prior biochemical studies in MEFs revealed that Taspase 1 cleaves MLL to transactivate Cyclin E [22]. However, MLL plays complex and context-dependent roles in cell cycle control. It can either positively or negatively regulate cell proliferation. For example, contrary to the positive regulation of cell proliferation that is more commonly observed, MLL was shown to negatively regulate pancreatic neuroendocrine cell proliferation. In this context MLL interacts with the tumor suppressor Menin to activate p27, a CDKI for cell cycle inhibition and thus prevent aberrant neoplastic proliferation [32]. Furthermore, an independently generated non-cleavable MLL mouse model showed 
no overt proliferation defects in MEFs [33]. Hence, it is of significance to address the role of MLL cleavage, if any, in $M M T V$-neu-driven breast tumorigenesis using genetic models. Our data suggested that specific ablation of Taspase1-mediated MLL cleavage could suppress $M M T V$-neu-driven breast cancer formation. We accordingly generated $M M T V-n e u ; M L L^{n c / n c}$ transgenic mice that harbor homozygous Taspase 1 non-cleavable (nc) alleles of $M L L$. Unlike Taspase $1^{-/}$mice, which died prematurely, $M L L^{n c / n c}$ mice were viable and fertile [34]. Like MMTV-neu;MMTV-cre;Tasp $1^{F /}$ mice (Figure 2A), most $M M T V-n e u ; M L L^{n c / n c}$ females (27 of 30) were, remarkably, free of breast cancer at 60 weeks of age (Figure $5 \mathrm{~A}$ ). We then determined whether $M L L^{n c / n c}$ females display any mammary gland defects. Whole mounts of $M L L^{n c / n c}$ female mammary glands at 6 weeks of age, day 13 of pregnancy, and day 1 of lactation did not reveal any abnormalities (Figure 5B). Altogether, these results indicate that Taspase1-mediated MLL cleavage plays a critical role in $M M T V$-neu-induced breast carcinogenesis in vivo.

Non-cleavable MLL disrupts MMTV-neu-induced aberrant proliferation and cyclin $E$ overexpression in mammary glands

We next investigated the molecular basis underlying $M L L^{n c / n c}$ females' resistance to $M M T V$-neu-induced breast tumorigenesis by comparing the mammary glands of $M M T V-n e u ; M L L^{n c h c}$ female virgin mice to those of wildtype and MMTV-neu;MMTV-cre;Tasp $1^{+/+}$mice at 12 and 20 weeks of age. Consistently, the increased duct and end bud density induced by the $M M T V$-neu transgene was not observed in mammary glands of MMTV-neu; $M$ $L L^{n c / n c}$ mice (Figure 6A). We subsequently performed immunohistochemistry analysis for histone $\mathrm{H} 3$ serine 10 phosphorylation ( $\mathrm{pH} 3 \mathrm{~S} 10)$ on the mammary glands of 12-week-old MMTV-neu; $M L L^{n c / n c}$ mice. Like MMTV$n e u ; M M T V-c r e ; \operatorname{Tasp}^{F /}$ and wild-type mice (Figure 4B), $M M T V$-neu; $M L L^{n c / n c}$ mice had 10 -fold fewer $\mathrm{pH} 3 \mathrm{~S} 10+$ cells in their mammary glands than $M M T V-n e u ; M M T V$ cre; Tasp $^{+/+}$mice (Figure 6B). Finally, we examined the expression of cyclins E, A, D, and CDKIs p16 and p27 in the mammary glands of 10-week-old MMTV-neu;MLL ${ }^{n c /}$ ${ }^{n c}$ mice. Non-cleavage of MLL suppressed MMTV-neuinduced cyclin E accumulation (Figure 6C), similar to what was observed in MMTV-neu;MMTV-cre;Tasp $1^{F /-}$ mice (Figure 4D). Overall, these genetic and biochemical results demonstrate that Taspase 1 and thus mature MLL $^{\mathrm{N} 320 / \mathrm{C} 180}$, generated upon Taspase1-mediated cleavage of the precursor MLL ${ }^{500}$, are required for MMTV-neudriven breast carcinogenesis, which involves transcriptional activation of Cyclin $E$ in the mammary glands.

\section{Discussion}

The cloning of Taspase1 (threonine aspartase) founded a novel class of endopeptidases that employ the NH2-terminal threonine of the mature $\beta$ subunit to cleave protein substrates after P1 aspartate [20]. In this study, using genetically well-defined mouse breast cancer models we show that Taspase 1 is required for MMTV-neu-driven mammary tumorigenesis, which represents the first in vivo study demonstrating that Taspase 1 ablation suppresses tumor initiation.

The mixed-lineage leukemia $(M L L)$ gene encodes an epigenetic transcriptional regulator belonging to the trithorax group family [35]. MLL is a confirmed substrate of Taspase1 [22]. In its best-known developmental role, MLL maintains proper expression of Hox genes and thus coordinates the segmental body plan of vertebrates $[36,37]$. Mice deficient for $M L L\left(M L L^{+/-}\right.$and $\left.M L L^{-/}\right)$accordingly display homeotic defects in their axial skeleton [37]. In addition to patterning body axis, MLL also regulates hematopoiesis, cell cycle, and cancer cell invasion [24, 37-42]. MLL possesses histone H3 lysine 4 (H3K4) methyl transferase (HMT) activity [43-46]. MLL-catalyzed $\mathrm{H} 3 \mathrm{~K} 4$ trimethylation (H3K4me3) activates transcription, leading to orchestrated upregulation of key developmental, cell cycle, and cancer cell invasion genes such as Hox genes, Cyclins E and A, and MMP1 and $M M P 3$, respectively [22, 24, 28, 45, 46]. Notably, the activity of MLL can be modulated by post-translational modifications, such as phosphorylation, ubiquitination, and proteolysis [20, 41, 42, 47]. The 500-kDa precursor MLL $\left(\right.$ MLL $^{500}$ ) undergoes Taspase1-mediated proteolytic cleavage, which gives rise to the mature MLL ${ }^{\mathrm{N} 320 / \mathrm{C} 180}$ heterodimer $[20,25]$ that binds to Cyclins $E$ and $A$ promoters $[22,28]$. MLL forms complexes with E2Fs to methylate $\mathrm{H} 3 \mathrm{~K} 4$ at promoters, and thereby transactivates $C y$ clins $E$ and $A[22,28]$. In the absence of Taspase1, MLL exists as MLL ${ }^{500}$, a noncleaved precursor with reduced HMT activity [20,22], and is unable to fully activate the expression of Cyclins E, $A$ and $B$ [22]. The in vivo significance of MLL cleavage has nonetheless been questioned on the grounds that whereas Taspase $1^{-/}$mice exhibit diverse developmental defects, $M L L^{n c / n c}$ mice are born at Mendelian ratio, fertile, and grossly normal [24, 33]. Our genetic study here unequivocally establishes the in vivo significance of the Taspase1-MLL-cyclin E pathway in carcinogenesis by demonstrating that MMTV-neu;M$L L^{n c / n c}$ mice are protected from MMTV-neu-driven breast cancer formation. Taspase1-mediated cleavage of MLL is therefore required for HER2/neu-induced tumorigenesis. However, whether cleavages of other Taspase 1 substrates, such as MLL2, have roles in tumorigenesis 


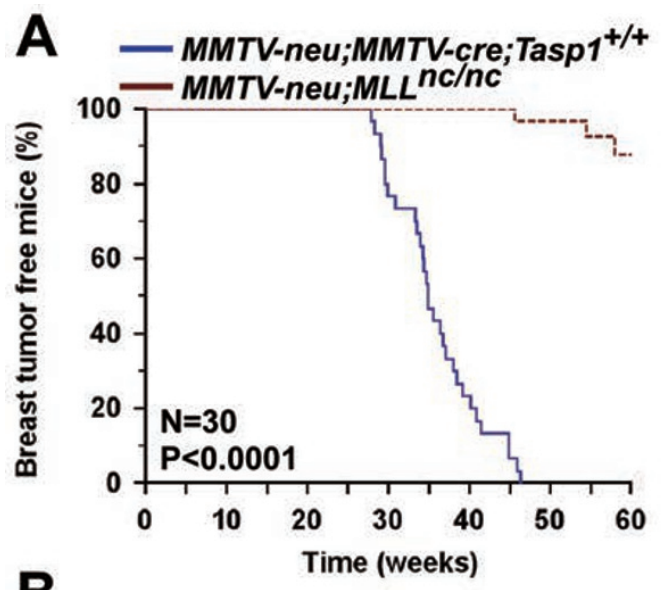

Figure 5 The cleavage of MLL by Taspase1 is required for MMTV-neu-driven breast cancer formation but dispensable for mammary gland development. (A) Kaplan-Meier curve of breast cancer incidence of MMTV-neu;MLL ${ }^{n / n c}$ mice. The same positive control MMTV-neu;MMTV-cre;Tasp ${ }^{+/+}$female mice (Figure 3) were plotted as control. $n=30$ mice per group. $P<0.0001$. (B) Whole mounts of female mammary glands of $M L L^{n c / n c}$ mice at the indicated age and gestation status. $n=3-4$ mice per group. Representative images are presented. Scale bar, $1 \mathrm{~mm}$.

B

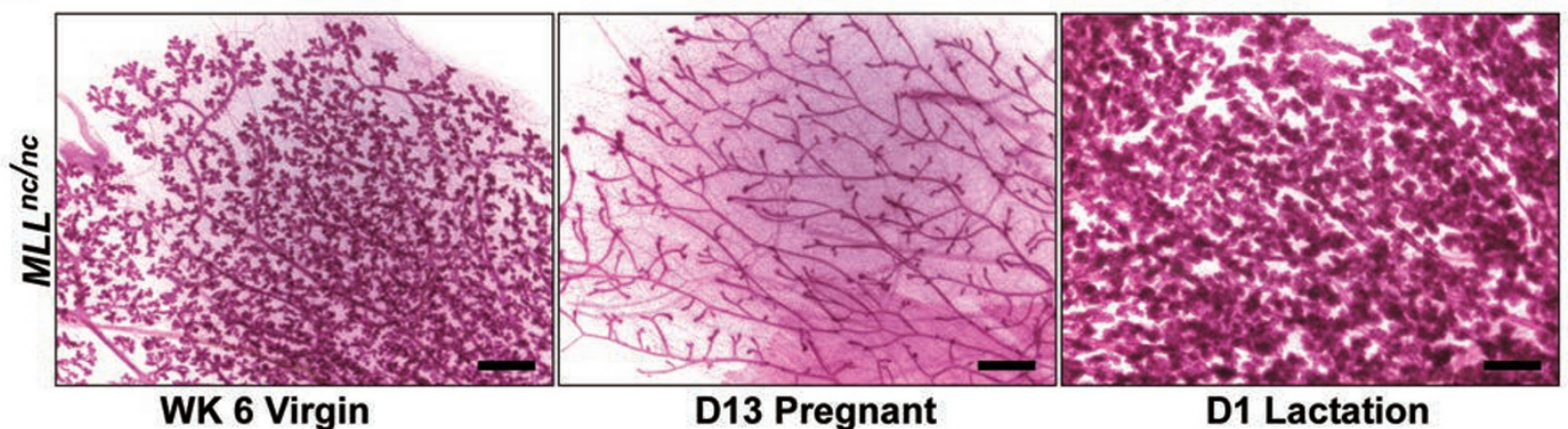

remains to be determined.

HER2-amplified/overexpressed breast cancer is characterized by an aggressive phenotype and poor overall survival. Although the application of anti-HER2 therapy has improved the clinical outcome of HER2-positive breast cancers, primary and secondary resistance constitute major obstacles to the further success of such treatment strategy. As cyclin E amplification/overexpression in HER2-positive human breast cancers results in decreased sensitivity to the anti-HER2 agent trastuzumab, high levels of cyclin E in human HER2-positive breast cancer may be predictive of resistance to anti-HER2 therapy $[7,16,17]$. Pharmacological inhibition of cyclin E expression may therefore benefit HER2-positive breast cancer patients by delaying disease progression and/or preventing recurrence while they are receiving anti-HER2 therapy. A series of studies including this current report clearly demonstrate that Taspase1 cleaves MLL to promote cyclin E expression and this Taspase1MLL-cyclin E axis is required for HER2/neu induced breast tumorigenesis [22, 26]. Therefore, administering Taspase1 inhibitors in conjunction with anti-HER2 agents could produce therapeutic benefits for HER2-positive breast cancer patients. As proteases are drug targets, small-molecule inhibitors of Taspase1 may be developed for cancer therapy.

Lines of evidence indicate the active participation of Taspase 1 in tumorigenesis, and thus support the development of small molecule inhibitors of Taspase 1 for potential cancer therapy. However, caution should be exercised while exploiting Taspase1 inhibition as a therapeutic means in treating human subjects. First and foremost, the severe perinatal lethality resulting from the embryonic loss of Taspase 1 suggests that inactivation of Taspase 1 by genetic or pharmacological means is inadvisable in pregnant females and children in order to avoid potential developmental sequelae [22]. Nevertheless, inactivation of Taspase 1 in fully developed adult mammals appears to be well-tolerated [29]. Cancer commonly hijacks key developmental pathways during tumorigenesis and thus frequently exhibits unique properties such as stem celllike and dedifferentiated states [48], which may underlie the preferential therapeutic benefit conferred by targeting Taspase 1 to treat cancers.

Primitive Taspase 1 inhibitors (TASPINs) were designed and discovered, lending support to developing highly effective, specific Taspase 1 inhibitors for cancer therapy [29]. Interestingly, although mammary glandspecific knockout of Taspasel disrupts MMTV-neudriven breast tumorigenesis, the same genetic deletion of 
A
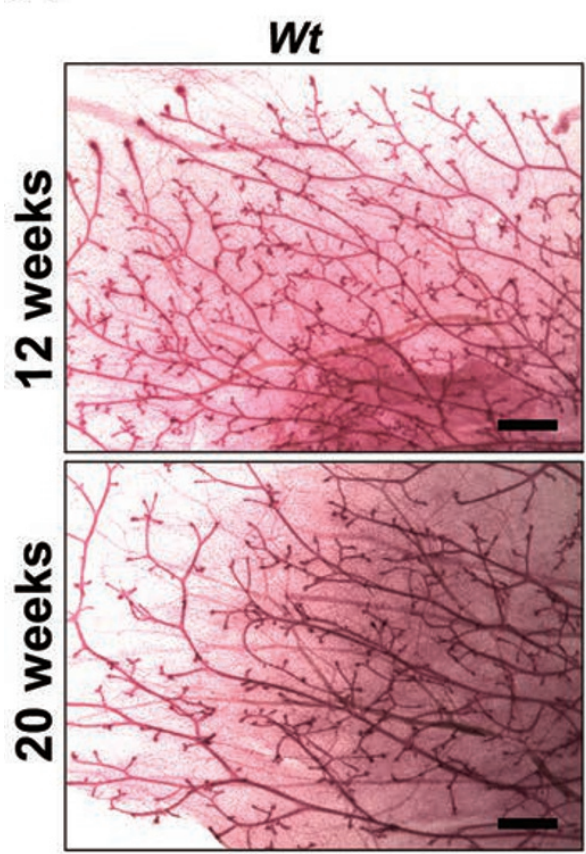

MMTV-neu;
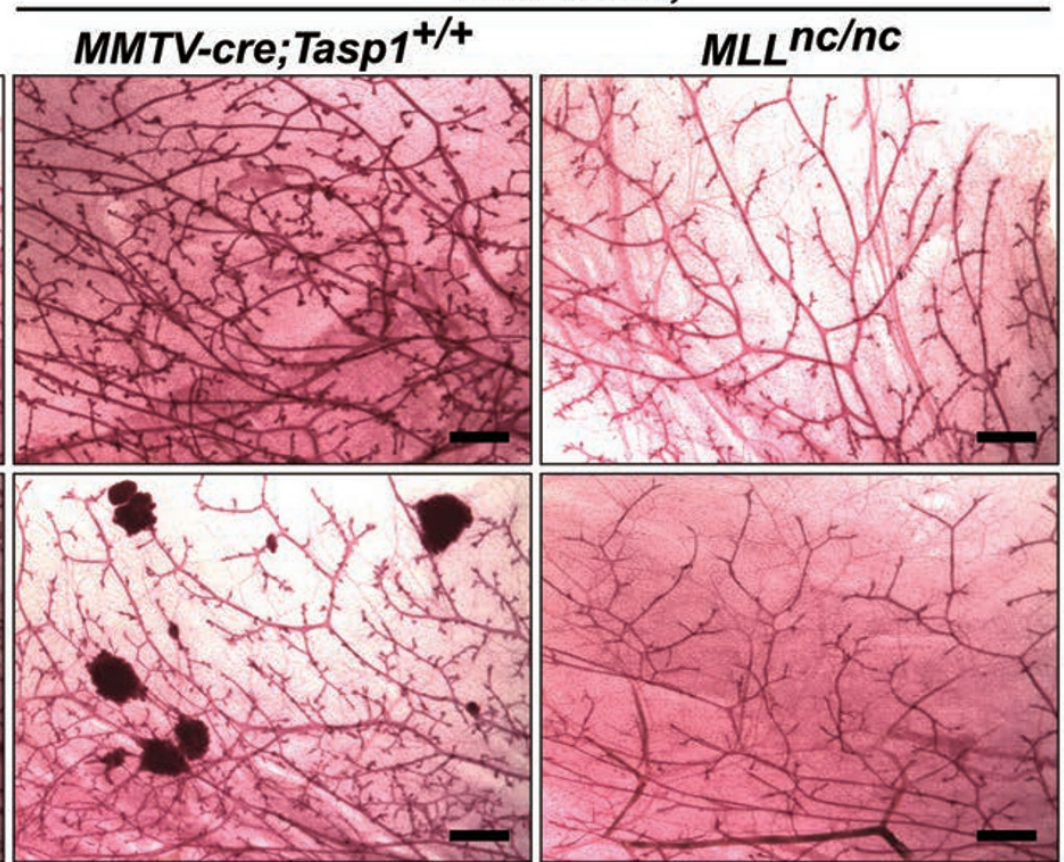

B

MMTV-neu;

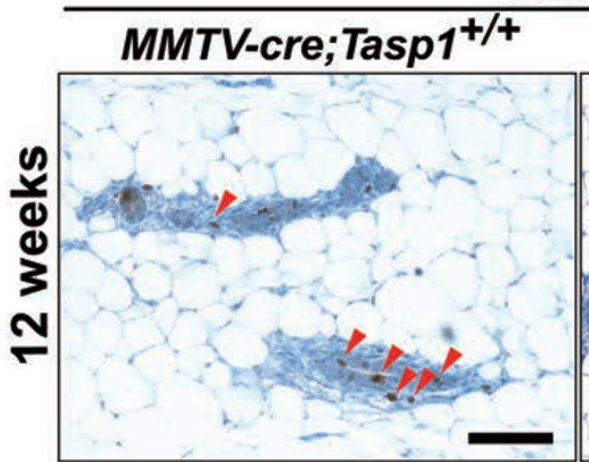

C

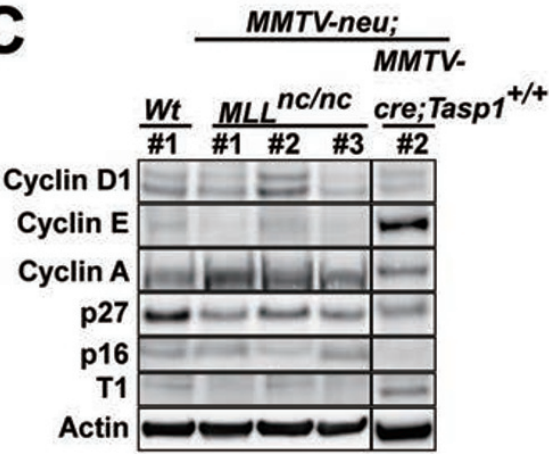

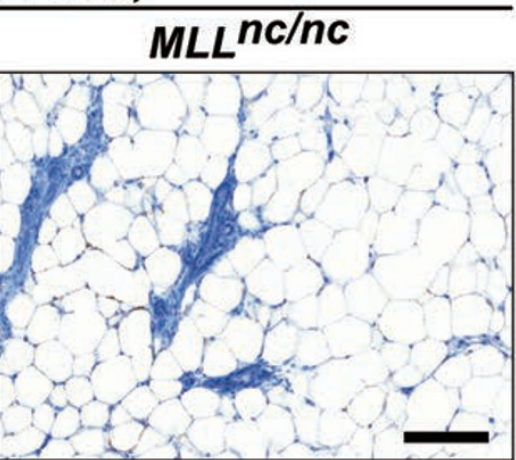

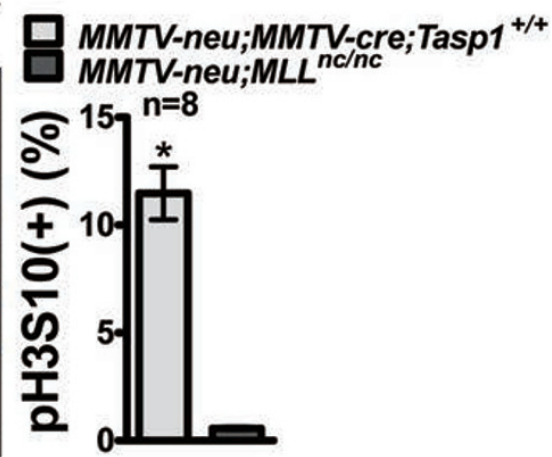

Figure 6 Non-cleavage of MLL disrupts tumor formation and induction of cyclin $\mathrm{E}$ in MMTV-neu mouse mammary glands. (A) Whole mounts of female mammary glands of the indicated genotypes at 12 and 20 weeks of age. $n=$ 5-15 mice per group. Representative images are presented. Scale bar, $1 \mathrm{~mm}$. (B) pH3S10 staining of female mammary glands of the indicated genotypes at 12 weeks of age. $n=8$ mammary glands per group. Representative images are presented. Red arrow head indicates positive cells. Scale bar, $100 \mu \mathrm{m}$. ${ }^{*} P$ $<0.0001$. (C) Western blot analyses of cyclins, CDKIs, and Taspase1 using female mammary glands and tumors of the indicated genotypes. Wt \#1 and MMTV-neu;MMTV-cre;Tasp $1^{+/+}$tumor \#2 extracts (as Figure 4D) serve as reference for relative abundance. $\beta$-Actin serves as loading control. 
Taspase1 in MMTV-wnt; $M M T V$-cre;Tasp $1^{F /}$ mice did not deter $M M T V$-wnt-driven breast carcinogenesis (Figure $2 \mathrm{C})$. These data highlight the various mechanisms underlying individual tumorigenesis and the importance of selecting responsive cancers that might benefit from the treatment with TASPINs. Although it is beyond the scope of this study, it is of our interests to discover biomarkers that are predictive of Taspase 1 addiction. Based on our unpublished data, Taspase1 has a very long protein half-life and is likely under multilayers of regulations in addition to transcription. Nevertheless, we analyzed the data of Taspase 1 expression in HER2-positive and -negative tumors from breast TCGA dataset, and did not observe significant differences in Taspase 1 expression (Supplementary information, Figure S5). Since Taspase1 is important in many aspects of cancer biology, Taspase 1 inhibitors likely will benefit patients with different cancer types. Further studies with regard to the involvement of Taspase 1 in various oncogenic pathways and the pathogenesis of subtypes of cancer could guide selection of cancer patients who would benefit from the inhibition of Taspase1.

\section{Materials and Methods}

\section{Animal studies}

All animal work was performed in accordance with MSKCC guidelines and IACUC approval. Mice were monitored for tumors by palpation twice a week. Tumor free Kaplan-Meier survival was calculated using MedCalc analysis software.

Cell culture, knockdown, proliferation, cell cycle, cell death, and western blot assays

BT-474 and HCC1419 cell lines were obtained from American Type Culture Collection and cultivated for no more than 2 months after each frozen aliquot was thawed. Amphotropic retrovirus carrying Taspase1 specific knockdown hairpin was generated as described $[26,29]$. To assay cell proliferation, $1 \times 10^{5}$ cells were seeded onto each well of a 6 -well plate and counted 4 days later. Cell cycle and cell death analyses were performed as described [26]. For western blot, cells and tissues were lysed in standard RIPA buffer. The anti-Taspase 1 rabbit polyclonal antibody is as described $[22,26]$. Antibodies for cyclin E2 (4132, Cell Signaling), cyclin A (C4710, Sigma), p21 (sc-397, Santa Cruz Biotechnology), p27(sc-528, Santa Cruz Biotechnology), cyclin D1(sc-450, Santa Cruz Biotechnology), p16(554079, BD Pharmingen), and ErbB2(OP-15, Calbiochem) were purchased from indicated companies. Antibodies were detected using the enhanced chemiluminescence method (Western Lightning, PerkinElmer). Immunoblot signals were acquired with the LAS-3000 Imaging system (FujiFilm) and were analyzed with ImageJ software.

\section{Knockdown resistant Taspase1}

Knockdown resistant Taspase1 (RT1) was generated by site-directed mutagenesis to create synonymous mutations at amino acids 387 to 392 (GGA AAG GCG AAA ACG CAT) of Taspase1. The
cDNA was inserted into an MSCV-neo expression construct.

\section{Soft agar assay}

$1 \times 10^{5}$ cells were seeded onto a $6 \mathrm{~cm}$ dish containing a top layer of $0.3 \%$ noble agar and a bottom layer of $0.6 \%$ noble agar base. Cells were fed with media every 3 days. After 3 weeks, colonies with diameter larger than $200 \mu \mathrm{m}$ were scored. Three independent triplicate experiments were performed.

\section{Quantitative RT-PCR}

mRNA was isolated using TRIzol (Invitrogen) and further purified using the RNeasy Mini Kit (Qiagen) according to the manufacturer's protocol. Reverse transcription reactions were carried out as described with Superscript II (Invitrogen) and random decamer primers (Ambion) [20, 22, 34]. Quantitative RT-PCR was performed using TaqMan $2 \times$ buffer and an ABI Prism 7300 sequence detection system (Applied Biosystems). For cyclin E1 and E2 reactions, the TaqMan Hs01026536 and Hs00180319 probes were used, and for cyclin A, SYBR Green master mix and the following primers were used: CAA AGC ACC ACA GCA TGC ACA AC and GAT TTA GTG TCT CTG GTG GGT TGA GG. All reactions were normalized against 18s rRNA using an 18s rRNA TaqMan probe (Applied Biosystems).

\section{Mammary gland whole-mount assays}

Mouse mammary glands were surgically dissected, spread onto a glass slide, and fixed in a 1:3:6 mixture of glacial acetic acid/ chloroform $/ 100 \%$ ethanol. Following hydration, they were stained overnight in $0.2 \%$ carmine and $0.5 \% \operatorname{ALK}\left(\mathrm{SO}_{4}\right)_{2}$; dehydrated in graded solutions of ethanol, cleared in xylenes; and mounted with Permount.

\section{Immunohistochemistry}

Tissue samples were fixed in 4\% paraformaldehyde and embedded in paraffin. Sections of 5- $\mu$ m thickness were prepared. pH3S10 was detected by immunohistochemistry using the antibody from Millipore (06-570).

\section{Statistical analysis}

Student's $t$-test was performed to compare means between two groups. Data were expressed as the mean $\pm \mathrm{SD}$ or \pm sem as indicated.

\section{Taspasel gene expression in human breast cancer}

To determine whether Taspase1 (Tasp1) expression differs in HER2 positive and HER2 negative human breast cancer, normalized RNA sequencing (RNA-Seq) data produced by The Cancer Genome Atlas (TCGA) [49] were downloaded from Broad GDAC Firehose. PAM50 subtype classifications were available for 500 of the 526 primary breast invasive carcinoma tumor samples TCGA subjected to mRNA expression profiling using the Illumina HiSeq 2000 RNA Sequencing Version 2 platform. The subtype classifications were obtained through cBioPortal for Cancer Genomics, and the 26 samples lacking classifications were discarded. The Tasp1 mRNA expression values of the remaining samples formed a dataset that was partitioned by HER2 status to form HER2 positive and HER2 negative datasets. Two-tailed Mann-Whitney tests were performed to compare the means of the HER2 positive and HER2 negative datasets. 


\section{Acknowledgments}

This study is supported by NIH R01 CA119008 to JH. We thank Dr Can G Pham for his expert editorial recommendation.

\section{References}

1 Baselga J. Treatment of HER2-overexpressing breast cancer. Ann Oncol 2010; 21 Suppl 7:vii36-vii40.

2 Olayioye MA, Neve RM, Lane HA, Hynes NE. The ErbB signaling network: receptor heterodimerization in development and cancer. EMBO J 2000; 19:3159-3167.

3 Citri A, Yarden Y. EGF-ERBB signalling: towards the systems level. Nat Rev Mol Cell Biol 2006; 7:505-516.

4 Higgins MJ, Baselga J. Targeted therapies for breast cancer. $J$ Clin Invest $2011 ; \mathbf{1 2 1}: 3797-3803$.

5 Slamon DJ, Clark GM, Wong SG, Levin WJ, Ullrich A, McGuire WL. Human breast cancer: correlation of relapse and survival with amplification of the HER-2/neu oncogene. Science 1987; 235:177-182.

6 Slamon DJ, Godolphin W, Jones LA, et al. Studies of the HER-2/neu proto-oncogene in human breast and ovarian cancer. Science 1989; 244:707-712.

7 Scaltriti M, Eichhorn PJ, Cortes J, et al. Cyclin E amplification/overexpression is a mechanism of trastuzumab resistance in HER2+ breast cancer patients. Proc Natl Acad Sci USA 2011; 108:3761-3766.

8 Baselga J, Swain SM. Novel anticancer targets: revisiting ERBB2 and discovering ERBB3. Nat Rev Cancer 2009; 9:463-475.

9 Marty M, Cognetti F, Maraninchi D, et al. Randomized phase II trial of the efficacy and safety of trastuzumab combined with docetaxel in patients with human epidermal growth factor receptor 2-positive metastatic breast cancer administered as first-line treatment: the M77001 study group. J Clin Oncol 2005; 23:4265-4274.

10 Hanahan D, Weinberg RA. The hallmarks of cancer. Cell 2000; 100:57-70.

11 Park MT, Lee SJ. Cell cycle and cancer. J Biochem Mol Biol 2003; 36:60-65.

12 Murray AW. Recycling the cell cycle: cyclins revisited. Cell 2004; 116:221-234.

13 Malumbres M, Barbacid M. Cell cycle, CDKs and cancer: a changing paradigm. Nat Rev Cancer 2009; 9:153-166.

14 Ohtsubo M, Theodoras AM, Schumacher J, Roberts JM, Pagano M. Human cyclin E, a nuclear protein essential for the G1-to-S phase transition. Mol Cell Biol 1995; 15:2612-2624.

15 Yu Q, Geng Y, Sicinski P. Specific protection against breast cancers by cyclin D1 ablation. Nature 2001; 411:1017-1021.

16 Mittendorf EA, Liu Y, Tucker SL, et al. A novel interaction between HER2/neu and cyclin E in breast cancer. Oncogene 2010; 29:3896-3907.

17 Keyomarsi K, Tucker SL, Buchholz TA, et al. Cyclin E and survival in patients with breast cancer. N Engl J Med 2002; 347:1566-1575.

18 Porter PL, Malone KE, Heagerty PJ, et al. Expression of cell-cycle regulators $\mathrm{p} 27 \mathrm{Kip} 1$ and cyclin $\mathrm{E}$, alone and in combination, correlate with survival in young breast cancer patients. Nat Med 1997; 3:222-225.
19 Hsieh JJ, Ernst P, Erdjument-Bromage H, Tempst P, Korsmeyer SJ. Proteolytic cleavage of MLL generates a complex of $\mathrm{N}$ - and C-terminal fragments that confers protein stability and subnuclear localization. Mol Cell Biol 2003; 23:186-194.

20 Hsieh JJ, Cheng EH, Korsmeyer SJ. Taspase1: a threonine aspartase required for cleavage of MLL and proper HOX gene expression. Cell 2003; 115:293-303.

21 Zhou H, Spicuglia S, Hsieh JJ, et al. Uncleaved TFIIA is a substrate for taspase 1 and active in transcription. Mol Cell Biol 2006; 26:2728-2735.

22 Takeda S, Chen DY, Westergard TD, et al. Proteolysis of MLL family proteins is essential for taspase1-orchestrated cell cycle progression. Genes Dev 2006; 20:2397-2409.

23 Capotosti F, Hsieh JJ, Herr W. Species selectivity of mixed-lineage leukemia/trithorax and HCF proteolytic maturation pathways. Mol Cell Biol 2007; 27:7063-7072.

24 Takeda S, Liu H, Sasagawa S, et al. HGF-MET signals via the MLL-ETS2 complex in hepatocellular carcinoma. J Clin Invest 2013; 123:3154-3165.

25 Khan JA, Dunn BM, Tong L. Crystal structure of human Taspase1, a crucial protease regulating the function of MLL. Structure 2005; 13:1443-1452.

26 Chen DY, Liu H, Takeda S, et al. Taspase1 functions as a non-oncogene addiction protease that coordinates cancer cell proliferation and apoptosis. Cancer Res 2010; 70:5358-5367.

27 Ohtani K, DeGregori J, Nevins JR. Regulation of the cyclin E gene by transcription factor E2F1. Proc Natl Acad Sci USA 1995; 92:12146-12150.

28 Tyagi S, Chabes AL, Wysocka J, Herr W. E2F activation of $\mathrm{S}$ phase promoters via association with HCF-1 and the MLL family of histone H3K4 methyltransferases. Mol Cell 2007; 27:107-119.

29 Chen DY, Lee Y, Van Tine BA, et al. A pharmacologic inhibitor of the protease Taspase 1 effectively inhibits breast and brain tumor growth. Cancer Res 2012; 72:736-746.

30 Lee JT, Chen DY, Yang Z, Ramos AD, Hsieh JJ, Bogyo M. Design, syntheses, and evaluation of Taspase1 inhibitors. Bioorg Med Chem Lett 2009; 19:5086-5090.

31 Muller WJ, Sinn E, Pattengale PK, Wallace R, Leder P. Single-step induction of mammary adenocarcinoma in transgenic mice bearing the activated c-neu oncogene. Cell 1988; 54:105-115.

32 Milne TA, Hughes CM, Lloyd R, et al. Menin and MLL cooperatively regulate expression of cyclin-dependent kinase inhibitors. Proc Natl Acad Sci USA 2005; 102:749-754.

33 Yokoyama A, Ficara F, Murphy MJ, et al. MLL becomes functional through intra-molecular interaction not by proteolytic processing. PLoS One 2013; 8: 73649.

34 Oyama T, Sasagawa S, Takeda S, et al. Cleavage of TFIIA by Taspase1 Activates TRF2-Specified Mammalian Male Germ Cell Programs. Dev Cell 2013; 27:188-200.

35 Martinez AM, Cavalli G. The role of polycomb group proteins in cell cycle regulation during development. Cell Cycle 2006; 5:1189-1197.

36 Krumlauf R. Hox genes in vertebrate development. Cell 1994; 78:191-201.

$37 \mathrm{Yu}$ BD, Hess JL, Horning SE, Brown GA, Korsmeyer SJ. Altered Hox expression and segmental identity in Mll-mutant mice. Nature 1995; 378:505-508. 
38 McMahon KA, Hiew SY, Hadjur S, et al. Mll has a critical role in fetal and adult hematopoietic stem cell self-renewal. Cell Stem Cell 2007; 1:338-345.

39 Hess JL, Yu BD, Li B, Hanson R, Korsmeyer SJ. Defects in yolk sac hematopoiesis in Mll-null embryos. Blood 1997; 90:1799-1806.

40 Jude CD, Climer L, Xu D, Artinger E, Fisher JK, Ernst P. Unique and independent roles for MLL in adult hematopoietic stem cells and progenitors. Cell Stem Cell 2007; 1:324-337.

41 Liu H, Takeda S, Cheng EH, Hsieh JJ. Biphasic MLL takes helm at cell cycle control: implications in human mixed lineage leukemia. Cell Cycle 2008; 7:428-435.

42 Liu H, Takeda S, Kumar R, et al. Phosphorylation of MLL by ATR is required for execution of mammalian S-phase checkpoint. Nature 2010; 467:343-346.

43 Krivtsov AV, Armstrong SA. MLL translocations, histone modifications and leukaemia stem-cell development. Nat Rev Cancer 2007; 7:823-833.

44 Liedtke M, Cleary ML. Therapeutic targeting of MLL. Blood 2009; 113:6061-6068.

45 Milne TA, Briggs SD, Brock HW, et al. MLL targets SET do- main methyltransferase activity to Hox gene promoters. Mol Cell 2002; 10:1107-1117.

46 Mohan M, Lin C, Guest E, Shilatifard A. Licensed to elongate: a molecular mechanism for MLL-based leukaemogenesis. Nat Rev Cancer 2010; 10:721-728.

47 Yokoyama A, Ficara F, Murphy MJ, et al. Proteolytically cleaved MLL subunits are susceptible to distinct degradation pathways. J Cell Sci 2011; 124:2208-2219.

48 Reya T, Morrison SJ, Clarke MF, Weissman IL. Stem cells, cancer, and cancer stem cells. Nature 2001; 414:105-111.

49 Comprehensive molecular portraits of human breast tumours. Nature 2012; 490:61-70.

(Supplementary information is linked to the online version of the paper on the Cell Research website.)

(c) (1) () $\odot$ This work is licensed under the Creative Commons Attribution-NonCommercial-No Derivative Works 3.0 Unported License. To view a copy of this license, visit http:// creativecommons.org/licenses/by-nc-nd/3.0 